Paper prepared for publication in the Heggenhougen, Kris (ed.) "Encyclopedia of Public Health" (forthcoming)

\title{
Provider Payment Methods and Incentives
}

\section{Randall P. Ellis and Michelle McKinnon Miller}

\author{
Boston University \\ Department of Economics \\ 270 Bay State Road \\ Boston, Massachusetts 02215 \\ USA
}

May 11, 2007

\section{Key Words}

Provider payment, fees schedules, Diagnosis Related Groups (DRG), moral hazard, selection, capitation, incentives, risk adjustment.

\begin{abstract}
Diverse provider payment systems create incentives that affect the quantity and quality of health care services provided. Payments can be based on provider characteristics, which tend to minimize incentives for quality and quantity. Or payments can be based on quantities of services provided and patient characteristics, which provide stronger incentives for quality and quantity. Payments methods using both broader bundles of services and larger numbers of payment categories are growing in prevalence. The recent innovation of performance-based payment attempts to target payments on key patient attributes so as to improve incentives, better manage patients, and control costs.
\end{abstract}


There are many ways that health care providers can be paid. In India, government physicians are paid a salary and in Canada physicians are generally paid according to a government-regulated fee schedule. In the Netherlands however, office-based physicians receive capitated payments for much of their revenue. Similar variations are seen in payments to hospitals, which may be paid using fixed budgets, detailed fee schedules, or episode-based systems. In this chapter we develop a conceptual framework for thinking about methods of provider payment, focusing on payments to primary care doctors and to acute care hospitals. Both the way payments are made and the market setting can affect the cost, quantity, and quality of care provided. Therefore, we also discuss how payments affect incentives. The final section discusses recent payment systems that reward selected performance measures.

The focus of this chapter is on payments made to providers by social health insurance, private health insurance, other health plans, governments or employers. Since these payments are not made by consumers, economists call them supply side payments. Demand side payments (often called cost sharing) are those made by consumers directly to providers. Cost sharing is discussed elsewhere in this volume. Clearly provider compensation and incentives are impacted by both supply and demand side payments, but this chapter focuses on supply side payments only.

We begin by considering four broad dimensions of provider payment. The first dimension is the type of information used to calculate payments. The second dimension is the breadth of provider payment: Are doctors and hospitals paid narrowly for their own services, or are they paid broadly for bundles of related services, such as laboratory tests and other provider services? The third dimension of payment is the coarseness of the payment classification system used: Are there relatively few payment categories or are there many? The fourth dimension is the generosity of the payments: Are payments low or high? We briefly consider each of these dimensions and how common payment systems utilize each element. After payment dimensions are introduced, we discuss the incentive properties of each method of payment.

\section{Information used for provider payment}

Every provider payment system can be characterized by the information used to calculate provider payments. This information can be based on provider characteristics, patient characteristics or the characteristics of the services provided. Each type of information can be conceptualized as forming a triangle, as displayed in Figure 1. Provider payments can either use information of one type as represented by the vertexes of the triangle, or payments can be based on hybrids of two or three types of information, as represented by the sides and interior of the triangle.

$<$ Figure 1 near here $>$

At one extreme, point A, payments only depend on the provider's own characteristics. For example, doctors can be paid a salary regardless of how many or which patients they see and regardless of what services they provide. These salaries may 
vary by provider characteristics, such as specialty, training or experience. In many countries, including India and South Africa, public sector doctors are paid a salary. Even in the United States, doctors working for the federal Veterans' Administration and doctors in certain staff-model health maintenance organizations are paid a salary. Similarly, in many countries hospitals receive "block grants;" block grants are budgets based on hospital size or type without explicit regard to the number or type of patients seen or the services provided. Many public hospitals, including those in Spain and France, receive fixed annual budgets. Such hospital payment systems invariably adjust for variables such as the numbers of beds or population served, but these are still more correctly thought of as features of the provider and their market rather than patient or service characteristics. For a useful discussion of France's fixed budget system, see Sauvignet (2005).

Another payment system is represented by point B in Figure 1, in which payments are based solely on the health services provided. Fee based payment systems and costbased payment systems each pay providers according to the quantity of services given. Payments do not depend on who provides the services or who receives them. Under a fee system providers are paid for each service provided and hence are often called fee-forservice payment. Fee payment systems can either allow the provider or the payer to set fee levels. Fee schedules set by the payer are the most common form of reimbursement for doctors; they are used in Canada, Germany, Norway, and the United States. Under a cost-based system, providers may be reimbursed at the end of the year for the cost of inputs (lab material, supplies, and staff salaries) regardless of how these inputs are used. In both cases, if providers use more resources to treat patients, revenues increase.

A third possibility (point $\mathrm{C}$ in Figure 1) is that provider payments are based exclusively on patient characteristics. A pure capitation system is a payment system based on patient characteristics. Under capitation, physicians receive a fixed amount of money for each patient they manage. Thus, a physician's gross income depends upon the total number of people he cares for; the physician's income does not depend on the type of provider administering the service, the type of service being provided, or the quantity of services provided. Physician capitation payments were made during the 1990s to general practitioners in the United Kingdom and are used today in the Netherlands (Exter et al., 2004). Similarly, hospitals can be paid according to a pure Diagnostic Related Grouping (pure DRG) payment system. Under a pure DRG payment system, a hospital receives a fixed amount determined solely by the patient's diagnostic group. Again, the hospital's revenue only depends on the patients' characteristics, not on the provider characteristics or the service characteristics.

Payment systems are not limited to the three cases corresponding to points A, B, and $\mathrm{C}$; providers can be compensated according to hybrids of different dimensions. Some systems are based on a hybrid of provider and service characteristics, corresponding to point D. The office physician payment system in Germany currently has features that make it appear this way. Although doctors receive fee-for-service reimbursement, the total of all such payments is subject to a cap which varies by provider specialty. The payment caps work much like a salary, while the fee schedule is based on 
services actually provided (Busse and Riesberg, 2004). Alternatively, provider characteristics influence payments when fee schedules vary with the provider's training or specialty. For example, in the US, a service provided by a doctor is often paid more highly than the same service provided by a nurse or other clinician. A different possibility arises when payment systems reflect not only what services are provided but also who receives them; this corresponds to point $\mathrm{E}$, a payment hybrid of patient and service characteristics. For example, provider fees schedules may vary by insurance plan. In the US, providers receive different compensation from patients with commercial insurance than from patients with Medicaid coverage. If the payment from commercial insurance exceeds the payment from Medicaid, and if a physician has enough business from commercially insured patients, then the physician has a reduced financial incentive to treat Medicaid patients. Similarly, in Germany, providers receive different compensation from patients in public versus private Sickness Funds. Finally, hybrids corresponding to point $\mathrm{F}$ are also possible, in which some compensation is based on both provider and patient characteristics.

Over the past two decades, payment mechanisms that combine provider characteristics, service characteristics, and patient characteristics have been developed. Payment systems combining all three of these dimensions are represented by point G. An example is the Diagnostic Related Grouping (DRG) payment system adopted in many countries, including the US. In 1983, the US Medicare program began using DRGs to standardize payments and help to control hospital costs. Although many adjustments are made, DRG payments are a fixed prices per admission, with the payments reflecting patient characteristics (their diagnoses and age), health services provided (procedure codes), and provider characteristics (type of hospital). Australia, Germany, and several other countries have customized DRGs to their own needs.

\section{Breadth of the payment system}

$<$ Figure 2 goes about here. $>$

Separate from the issue of the information used to determine the payment system, is the issue of how broadly or narrowly provider services are aggregated. Under a narrow system, doctors are paid explicitly for each procedure provided and separate payments are made for each laboratory test or procedure done by other providers. A broader payment system might bundle jointly performed procedures into one fee. A system might broaden even further to include associated fees for laboratory services. A still broader system might make payments to a doctor that includes the cost of specialists, forcing the doctor to internalize the cost of all physician services.

Similar issues of the breadth of the provider payment system arise with hospital payment. Hospital DRG or per diem rates can narrowly cover only the room and board cost, or may include nursing services. Even more broadly, they may include hospital physician services. In the US, certain DRG payments have even been broadened to include the cost of services provided after discharge. In some countries hospitals are even given responsibility for certain kinds of primary care services. 
This breadth of payments is often discussed in terms of "bundling" of services. Recent trends in the US have been toward increased bundling of payments. Similar innovations are being tried in many other countries.

\section{Fineness of the payment system}

How many payment categories should be used? Should there be many gradations in payment (a "fine" system), or is simpler ("coarse") system appropriate? In countries such as Taiwan and Korea, physician fee schedules are fairly coarse, with very few fee categories, while physician fee schedules in Australia and the US are very fine, with many gradations in fee levels. In 2004, Germany reduced the fineness of its fee system.

$<$ Figure 3 goes about here. $>$

Similar issues of coarseness and fineness arise with DRGs, capitation, and physician salaries. In the US and many other countries, the DRG and capitation systems have become finer and finer, with increased distinctions made in payments. Coarse or fine gradations in payments are possible with each payment system.

The optimal fineness of the payment system reflects a tradeoff between the potentially improved fairness and reduced selection incentives resulting from a fine system and the challenges of monitoring and administering a fine system. Coarser systems should in principle be easier to monitor. If the payer cannot easily distinguish what service was actually provided, or the patient characteristics are not readily observable, then it may not be worthwhile to make such distinctions for payment.

\section{Generosity of payments}

Not only is the unit of payment important, but so is the overall generosity or level of payment. Empirical research shows that relative prices as well as their levels matter. If payment levels to providers are set too low, this reduces the incentive for quality for almost any payment system. We discuss this further below under incentives.

\section{Markets, provider competition, and the delivery structure}

By defining what services, patients, and providers are eligible for payment, a provider payment system also inherently defines the degree of competitiveness in the market as well as the market boundaries. If the physician payments restrict which patients or services a doctor will be reimbursed for treating, this has important implications for incentives. Similarly, incentives are affected if some but not all hospitals receive DRG payments. A careful summary of all of the different ways of organizing health care delivery markets lies well beyond the scope of this chapter, but we mention this topic now since the delivery structure is important in determining incentives, costs, and outcomes. 


\section{Incentives}

The above dimensions - the information used, the breadth, the fineness, the level of payments, and the market structure - all influence the incentives facing providers. Economists spend a great deal of time studying provider incentives because they generally believe the empirical evidence that providers respond to these incentives. In this section we describe the incentive effects of different payment systems commonly used around the world.

\section{Incentives created by salaries and fixed budgets}

Perhaps the easiest incentives to describe are those of salaried or fixed payment systems, where only provider information is used to calculate payments. Because revenues are fixed regardless of the volume of care rendered, physicians and hospitals have a financial incentive to inappropriately keep costs and costly effort at levels that are too low. Fixed revenues can also motivate physicians and hospitals to preferentially treat low risk patients, to try to avoid high cost patients, to make too many referrals, to minimize the number or intensity of services provided, and in general to underprovide quality. The one countervailing force is the possible threat to lose ones job, but countries that rely on salaried payments rarely fire salaried employees. Peer review and payer monitoring may also be effective at promoting quality. Payments based solely on provider characteristics do not tend to reward coordination of care across providers. Salaried or fixed hospital budget systems rely on provider altruism to ensure that appropriate and high quality services are provided. However, if providers realize costsavings from preventive care, these payment systems may encourage providers to engage in preventive efforts.

\section{Incentives created by fee-for-service}

The incentives of fee based payments are very different from those of salaried or capitated payments. Newhouse (2002) nicely summarizes the conventional economists' and policymakers' views that fee-for-service payment mechanisms create poor incentives for controlling cost or quantity. Fee-for-service rewards physicians with more revenue for rendering more services, whether or not these services improve the health or wellbeing of the patient. Under fee-for-service reimbursement, services that have little or no value to the consumer may be provided merely because they increase provider net income. Overprovision is likely to be a problem if the fee for a particular service is more than the incremental cost of that service to the provider. Carrin and Hanvoravongchai (2003) mention several instances in which fee-for-service payment systems caused the overprovision of services and thus caused a country's health care costs to rise. For example in 1987, when general practitioners in Copenhagen began to receive fees for some services, the provision of those services increased significantly. However, with fee-for-service, underprovision may occur if fees are too low relative to costs. For similar reasons, services not reimbursed through fees, such as preventative care counseling to the consumer (e.g., trying to change a patient's lifestyle or institute a smoking cessation 
program) receive little or no physician effort. Competition and consumer information, also affect incentives of fee based payments.

\section{Incentives created by pure DRGs and capitation}

Under pure DRGs, hospitals have a financial incentive to discharge a patient early since they will not be reimbursed for the additional costs they incur on behalf of the patient. If payments are relatively generous, then hospitals may compete to attract more patients. On the other hand, hospitals may discourage patients with less generous payments. DRGs also create incentives to game the system; providers may upcode, or classify patients into a higher DRG category, in order to receive a higher payment.

Capitation payments give physicians a fixed amount of money per patient, which creates a financial incentive to reduce costs per patient. Rather than necessarily managing care, capitation may instead result in providers competing to attract or select low risk patients, using referrals, or providing preventative care. Here, how competition is regulated will clearly matter. Capitation creates an incentive to potentially use low cost providers, increase outpatient services, reduce hospital admissions, and reduce days per admission. In addition, capitation creates incentives for the recipient of the capitated payment to increase the use of lower cost alternative providers such as nurse practitioners and physician assistants, rather than physicians.

\section{Incentives created by mixed payment systems}

An alternative to pure DRGs and capitated payments is the mixed payment system originally developed in Ellis and McGuire (1986). Under Ellis and McGuire's payment system, hospital payments are based on both diagnoses and the level of services provided. The great attraction of a mixed payment system is that it can reward services somewhat, but not as excessively as a fee-for-service payment. Partially using patient-based payments can reward providers for treating higher risk patients and overcome provider incentives to select only the most profitable, easier to treat patients. The Netherlands has implemented a form of the Ellis and McGuire mixed payment system in that office based doctors are paid partially on a fee basis and partially by capitation (Exter et al 2004).

\section{$\underline{\text { Incentives and fineness }}$}

The fineness of the payment system also affects provider incentives. A finer payment system tends to reduce cost variation within a payment category. This tends to create fewer incentives to select low cost patients. However, with more classifications, there are greater monitoring problems and more incentives to game the system. Providers may upcode, or classify patients into a higher payment category, in order to receive a larger compensation. In the literature, this is often referred to as "DRG creep" when referring to hospitals and "code inflation" when referring to procedure codes. Finer systems will tend to be more difficult to monitor since more patients will be near the margin where upcoding can make a difference. 
How coarseness/fineness and breadth/narrowness affect incentives is particularly apparent when payments go from physician fee schedules to DRG payments. This was illustrated in Korea when inpatient physicians switched from a relatively fine and narrow payment formula to a coarse and broad system. Until 1997, under the national health insurance program, a FFS payment system had been in place for approximately twenty years. The FFS payment system had led to a high volume of relatively low intensity health care, characterized by frequent but short visits and hospital stays. Under the FFS system, inpatient physicians had financial incentives to choose treatments with greater profits, and hence extensively used medical supplies and pharmaceuticals and avoided hospitalizations. In 1997, in hopes of controlling quantity and selection, a DRG pilot program was introduced for voluntarily participating providers. By its third year, the pilot program covered nine disease categories with twenty five DRG codes which depended on the severity and age of the patient. The program began with disease groups that had low expenditure variation, little disagreement among providers on treatment methods, low degree of uncertainty about treatment outcomes, high frequency of utilization, and lower possibility of DRG creep. Within each of these DRGs there were three types of patients: normal case, outlier below the lower-limit, and outlier above the upper-limit. The pilot program succeeded in lowering expenditure on medical care, reducing length of stay, and the reducing the use of antibiotics. Early evidence suggests that the program did not have a negative effect on the quality of care as measured by complications and re-operations (Kwon 2003).

The effects of incentives were also demonstrated when the National Health Insurance was established in Taiwan in 1995. At this time, Taiwan switched from paying office based doctors a salary to paying them according to a FFS schedule. Under FFS, a relatively coarse system of payment is used, based on a national fee schedule. The switch from salary to FFS was accompanied by an increase in the volume of services with shortened average visit length. According to one source, this led to misdiagnosis, improper treatment, and delays; the government responded by changing the fee structure so as to try to limit the number of patients each provider can treat during a given day (Cheng 2003).

The US Medicare system compensates providers according to a payment system that is finer (has more payment categories) than the systems in Korea and Taiwan. Under the US Medicare system, hospitals receive a prospectively determined price depending upon the patient's DRG. In 2006, the US Medicare system had 559 DRGs. As mentioned above, providers have a financial incentive to select the most profitable patients within one DRG group. Even with numerous DRGs, Dranove (1987) discusses the large differences in costs that occur within one DRG in the US Medicare system. In three Chicago area hospitals Dranove (1987) finds that one-sixth of the DRGs have a standard deviation that exceeds the mean. Large differences in the cost of treating patients classified in one DRG encourage hospitals to prefer treating less costly patients within a DRG. Thus, Dranove believes that a finer payment system is needed in order to avoid selection. One might worry that a finer US Medicare system would create more incentives to upcode patients. The classic study of this phenomenon in the US by Carter 
et al. (1990) found that upcoding or DRG creep accounted for less than one-third of the change in Medicare's Case Mix Index between 1986 and 1987.

Australia also uses a fine payment system to fund its public hospitals; the Australian National Diagnostic Related Groups has 667 categories (Hilless and Healy, 2001). In January 2004, Germany implemented a fine DRG system to compensate hospitals. The German DRGs take the diagnosis, severity, patient's age, and the intervention performed into account. In 2005, there were 878 DRGs in Germany. Because the payment systems in Australia and Germany are relatively fine, there are fewer opportunities for providers to select low cost patients. On the other hand, there are more opportunities for providers to upcode patients so as to increase payments.

\section{Performance-based payment systems}

In recent years there has been a great deal of interest in payment systems that reward performance. For example, doctors may receive bonuses based on performance targets, such as high immunization rates, or low surgical complication rates. Other payment systems reward providers according to how well they perform relative to their peers on various cost or quality measures. In the US, this has come to be called "pay for performance," often abbreviated P4P. P4P payment systems are commonly used by HMOs (Rosenthal et al 2006). P4P systems calculate payments on patient characteristics, for broad sets of providers, using potentially fine (not coarse) distinctions among patient types.

There is mixed evidence on the impact of these performance-based payment systems. A recent study by Rosenthal et al. (2005) and a review by Dudley and Rosenthal (2006) provide up-to-date discussions of the challenges in the US from using P4P. Large demonstrations that are in progress in the US and UK will also shed light on this new initiative.

There are many forms of P4P systems. Typically, primary care physicians are grouped into risk pools that share financial rewards and penalties; the size of the risk pool will influence a physician's responsiveness to incentives. Sometimes bonuses are shared among a large pool of physicians; in other cases individual physicians are eligible for incentive payments. The size of the risk pool affects the intensity of incentives as well as the financial risk born by providers.

Performance-based payment attempts to overcome the problem whereby doctors who provide inadequate care receive the same compensation as doctors who provide excellent care. Proponents of these systems argue that instead of paying doctors according to their characteristics, their patients' characteristics, or the type of service they provide, physicians should be paid according to their performance. Many believe that physicians will respond to performance incentives by providing higher quality care.

For some medical conditions, such as diabetes and asthma, there are easily recognizable quality measures which gauge provider performance. For example, if 
bonuses are allocated to providers who track diabetics' blood sugar levels, then doctors will be more likely to have diabetic patient's blood sugar monitored. Similarly, asthmatic patients will receive high quality treatment if physicians are given bonuses for prescribing the correct asthma medication. Performance based payment can encourage providers to give high quality care to patients with these two conditions.

However, there are challenges to performance based payment systems. First, opponents argue that these programs increase selection. Because physician ratings are based on claims data, their ratings may not fully reflect a patient's risk factors. If so, bonuses create incentives for doctors to drop risky patients within a payment category. Many doctors oppose performance-based payment systems because their payments rely too heavily on their patients' risk factors as well as their patients' actions (e.g., whether their patients take their prescription or return for a follow up appointment). Another concern is that only a relatively small number of performance measures are typically used. Incentives that do well on these measures may not carry over to other actions that are not measured or rewarded.

One widely cited study examined doctors who were offered bonuses if they complied with basic public health guidelines, including guidelines on preventative care. In 2003, California doctors were evaluated according to levels of breast-cancer screening, cervical cancer screening, and hemoglobin testing. The top rated doctors split a bonus pool of \$3.4 million. Compared to doctors in Oregon and Washington who were not offered bonuses, California doctors offered more cervical cancer screening. Although the quality of care increased in all three areas, only for cervical cancer screening was the improvement greater in California than in Oregon and Washington; this may have been because the financial rewards to quality were too low or because substantial quality improvements take time. Physician groups whose performance was initially the lowest improved the most, whereas physician groups who had previously achieved the targeted level of performance improved the least (Rosenthal et al 2005).

Bokhour et al. (2006) investigated the impact of P4P systems implemented in Massachusetts. This qualitative study interviewed 28 practice executives who noted that physicians viewed quality incentives as more aligned with their natural tendency to provide good quality of care. The study revealed that physicians appear to be motivated more by professional standards of quality than financial incentives.

P4P measures have also been introduced in other countries. Since 1990, general practitioners in the United Kingdom have commonly been paid according to targets. Initially, narrowly defined P4P target payments remunerated general practitioners if they delivered a minimum predetermined level of services or care. Kouides et al. (1998) found that immunization rates rose 5.9 percent compared to a control group when PCPs received an additional ten or twenty percent payment per shot for each immunization made over target rates. Ritchie et al. (1992) studied the effects of a lump sum payment received if a PCP immunized seventy percent of the eligible pre-school population. However, according to their study, the target payment intervention did not have a significant impact on immunization rates. In 2004, the United Kingdom introduced a 
large pay for performance contract for family practitioners. This $\mathrm{P} 4 \mathrm{P}$ payment system was much wider; with 146 quality indicators covering clinical care for 10 chronic diseases, organization of care, and patient experience. The National Health Service committed $£ 1.8$ billion ( $\$ 3.2$ billion) in additional funds for a three year period; the program was intended to increase family practitioners' income by up to 25 percent. After the first year, the median reported achievement was 83.4 percent (Doran et al 2006).

In Haiti, nongovernment organizations (NGOs) provide basic health care services including immunizations and prenatal care. Historically, these organizations were fully compensated for all their costs and therefore, they were not accountable for performance. In 1999, providers began operating under a performance based payment system. Under this new system, a portion of each NGO's historical budget was withheld. Physicians could earn back the amount withheld plus a bonus if they met specific targets including a targeted ten percent increase in children vaccinations. After the first year, the most striking result was the increase in immunization coverage in the NGO service areas. The doctors said that the shift in payment structure inspired them to question their model of service delivery; the possibility of earning bonuses sharpened their focus to achieving goals (Eichler et al 2001).

In 1998, the Cambodian government began an experiment which provided additional financial incentives for some health care workers based on performance. Historically in Cambodia, government health care providers received insufficient and irregular salaries, forcing them to seek alternative sources of income. Since this insufficient payment did not depend on performance, health facilities in Cambodia performed poorly. As detailed by Soeters and Griffiths (2003), after three years of implementation, when combined with monitoring, pay-for-performance improved utilization of health services and decreased total family health expenditure.

Similarly, a new output-based payment system was implemented in the Kabutare district of Rwanda in 2003. Before the introduction of the performance initiative, staff members received a fixed bonus in addition to their salaries. Under the new payment system, individuals kept their base salaries but an output-based remuneration replaced the fixed-bonus system. Meessen et al. (2007) found that productivity sharply increased between 2001 and 2003; average individual productivity increased by 53 percent under the new system.

There is evidence that performance payments need to be carefully designed and meaningful in order to have an impact. Hillman et al. (1998) could not reject that small financial bonuses did not affect compliance with cancer screening guidelines for a group of Medicaid physicians in Philadelphia. The authors attribute the insignificant result to the lack of awareness of the payments among physicians and to the difficulty of affecting treatment protocols when physicians have multiple payers.

At present the evidence is mixed on the effectiveness of pay for performance on quality and quantity of care. The theoretical and empirical research literature has not kept up with recent innovations, and many innovations are still being implemented that have 
not yet been validated or the incentives modeled. This remains an area promising possibly dramatic advances and worthy of significant new research.

\section{Trends for the future}

This chapter has shown that provider payment systems vary in a number of dimensions, all of which deserve consideration when considering reforms or study. Incentives created by provider payment vary according to each of these dimensions. Market characteristics, including provider competition, also influence incentives and outcomes in provider markets.

Other authors have noted recent trends in provider payment systems. Many countries have moved away from salary and fixed budget systems, which only reflect provider characteristics, and towards service-based and patient-based payment systems. Because fee-for-service payments may lead to the overprovision of services, sophisticated payers are using capitation or blends of different information so as to control costs.

Many countries are making their payment formulas finer, with more gradations of payment categories, and broader, with payments being made to few providers, who are in turn asked to manage or monitor other providers more carefully than an independent payer is able to. Trends towards pay-for-performance, with finely defined categories of information and broadly inclusive payments, reflect the latest movement toward patientbased payments. While the evidence is mixed about whether pay for performance will ultimately be widely adopted, the desire to create incentives for cost effective, high quality, accessible care suggests that elements of this approach will remain part of the provider payment strategy in the future. 


\section{Suggestions for further reading}

Carrin, G. and Hanvoravongchai, P. (2003). Provider payments and patient charges as policy tools for cost containment: how successful are they in high-income countries? Human Resources for Health 1(6), 1-20.

Jegers M., Kesteloot, K., De Graeve, D., and Gilles W, (2002) A typology for provider payment systems in health care. Health Policy 60 255-273.

McGuire, T.G. (2000). Physician Agency in A.J. Culyer A.J., and Newhouse, J.P. (eds.) Handbook in Health Economics, North Holland. pp. 461-536.

\section{References}

Bokhour, B.G. et al. (2006). Incentive implementation in physician practices: a qualitative study of practice executive perspectives on pay for performance. Medical Care Research and Review 63, 73S-95S.

Busse, R. and Riesberg, A. (2004). Health care systems in transition: Germany. Copenhagen: European Observatory of Health Care Systems.

Carrin, G. and Hanvoravongchai, P. (2003). Provider payments and patient charges as policy tools for cost containment: how successful are they in high-income countries? Human Resources for Health 1(6), 1-20.

Carter, G., Newhouse, J., and Relles, D. (1990). How much change in the case mix index is DRG creep? Journal of Health Economics 9, 411-428.

Cheng, T. (2003). Taiwan's new National Health Insurance program: genesis and experience so far. Health Affairs 22, 61-76.

Doran, T. et al. (2006). Pay-for-performance programs in family practices in the United Kingdom. The New England Journal of Medicine 355, 375-384.

Dranove, D. (1987). Rate-setting by diagnosis related groups and hospital specialization. The RAND Journal of Economics 18(3), 417-427.

Dudley, R.A., and Rosenthal M.B. (2006) Pay for Performance: A Decision Guide for Purchasers. Report prepared for the Agency for Healthcare Research and Quality, U.S. Department of Health and Human Services Contract No./Assignment No. 290-01-0001/298 AHRQ Publication No. 06-0047, April.

Eichler, R., Auxila, P., and Pollock, J. (2001) Promoting preventive health care: Paying for performance in Haiti. In Brook, P.J., and Smith, S.M. (eds.). Contracting for public services: Output-based aid and its applications. pp 65-71. World Bank Publications.

Ellis, R.P., and McGuire, T.G. (1986). Provider behavior under prospective reimbursement: cost sharing and supply. Journal of Health Economics 5, 129-152.

Exter A, Hermans H, Dosljak M, Busse R. (2004) Health care systems in transition: Netherlands. Copenhagen, WHO Regional Office for Europe on behalf of the European Observatory on Health Systems and Policies.

Hilless, M. and Healy, J. (2001). Health care system in transition: Australia. Copenhaugen: European Observatory on Health Care Systems.

Hillman, A.L. et al. (1998). Physician financial incentives and feedback: Failure to increase cancer screening in Medicaid managed care. American Journal of Public Health 88 (11), 1699-1701. 
Kouides, R.W. et al. (1998). Performance-based physician reimbursement and influenza immunization rates in the elderly. American Journal of Preventative Medicine 14, 89-95.

Kwon, S. (2003). Payment system reform for health care providers in Korea. Health Policy and Planning 18(1), 84-92.

Newhouse, J.P. (2002). Pricing the Priceless: A Health Care Conundrum. MIT Press.

Ritchie, L.D., Bisset, A.F., Russell, D., Leslie, V., and Thomson, I. (1992) Primary preschool immunization in Grampian: Progress and the 1990 contract. British Medical Journal 304(6830), 816-819.

Rosenthal, M.B. et al. (2006). Pay for performance in commercial HMOs. The New England Journal of Medicine 355, 1895-1902.

Rosenthal, M.B., Frank, R.G., Li, Z., and Epstein, A.M. (2005) Early evidence with payfor-performance: From concept to practice. The Journal of American Medical Association 294, 1788-1793.

Sauvignet, E. (2005) Le Financement du système de santé en France, WHO/EIP/HSF Discussion Paper, nr 1, pp.75-80.

Soeters, R. and Griffiths, F. (2003). Improving government health services through contract management: a case from Cambodia. Health Policy and Planning 18, 7483. 
Figure 1: Three Types of Information Available for Provider Payment

\section{Patient Characteristics}

MDs: physician capitation

Hospitals: pure DRGs

C

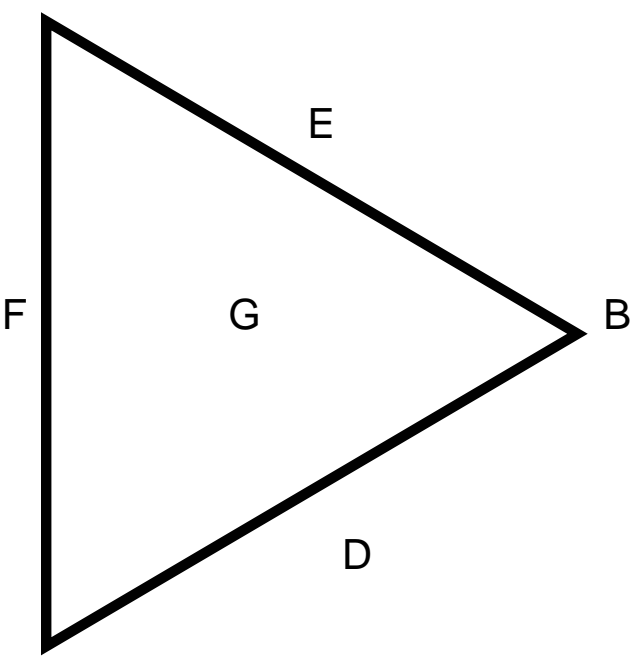

Service Characteristics

MDs: fees

Hospitals: fees

A

\section{Provider Characteristics}

MDs: salary

Hospitals: fixed budgets/block grants 
Figure 2: Breadth of Provider Payment ("bundling")

\begin{tabular}{|c|c|c|c|}
\hline & Narrow & & Broad \\
\hline MDs: & own time & lab tests & $\begin{array}{l}\text { other } \\
\text { specialists }\end{array}$ \\
\hline Hospitals: & $\begin{array}{l}\text { room and } \\
\text { board }\end{array}$ & $\begin{array}{l}\text { MD } \\
\text { inpatient } \\
\text { services }\end{array}$ & $\begin{array}{l}\text { related } \\
\text { outpatient } \\
\text { services }\end{array}$ \\
\hline
\end{tabular}

Figure 3: Fineness of Provider Payment (number of payment categories)

Coarse

Fine

MDs: $\quad$ fixed fee per office visit

uniform salary per MD

Capitation using age/gender

Hospitals: simple per diem rate

100 DRGs fees vary by treatment intensity or duration

salaries adjusted by specialty or age

capitation using age, gender, diagnoses

per diem varies by patient, department or service

800 DRGs 